\title{
A Complex Equivalent Source Method for Scattering Effect of Aircraft Noise
}

\author{
Yu Hou ${ }^{1}$ Xin Zhang $^{2}$ and David Angland ${ }^{3}$ \\ Faculty of Engineering and the Environment, \\ University of Southampton, Southampton, SO16 7QF, United Kingdom
}

\begin{abstract}
An equivalent source method using equivalent monopoles in complex space is developed for calculating scattering effects for aircraft noise applications. The new method overcomes deficiencies in traditional equivalent real space source methods in treating scattering off thin sharp edges. While a traditional equivalent source method may fail in situations where either thin objects and/or objects with sharp edges exist, e.g. wings, the new method is able to deal with both bluff bodies and wings with relatively small thickness and sharp trailing edges. In this work, we describe the method in detail. Verification and assessment of capabilities of the method are performed using benchmark test cases.
\end{abstract}

Keywords: complex equivalent source method, acoustic scattering, aircraft noise

\section{Nomenclature}

\author{
Aspect ratio \\ Ambient speed of sound $[\mathrm{m} /$ \\ Distance from scattering surface to (real space projection of) equivalent source surface \\ Frequency [Hz] \\ Green's function \\ Wavenumber $[1 / \mathrm{m}]$ \\ Freestream Mach number vector \\ Non-dimensional rotation speed of sources \\ Number of control points on scattering surface \\ Number of equivalent sources \\ Normal vector at control point with index i \\ Acoustic pressure in frequency domain $[\mathrm{Pa}]$ \\ Point per wavelength \\ Strength of equivalent source \\ Phase radius $[\mathrm{m}]$ \\ Amplitude radius [m] \\ Scaling factor for imaginary part of a complex equivalent monopole's location \\ Scaling factor for real part of a complex equivalent monopole's location \\ Free stream velocity vector \\ Volume of scattering object \\ Observer's location \\ Location of control point with index $\mathrm{i}$
}

\footnotetext{
${ }^{1}$ PhD student, Airbus Noise Technology Centre, Engineering Centre of Excellence, yh25g11@soton.ac.uk.

2 Airbus Professor of Aircraft Engineering, Airbus Noise Technology Centre, Engineering Centre of Excellence, Associate Fellow AIAA, xzhang@soton.ac.uk.

${ }^{3}$ Lecturer, Engineering Centre of Excellence, Member AIAA.
} 
Location of complex monopole

Location of equivalent source with index

Impedance $[\mathrm{Pa} \cdot \mathrm{s} / \mathrm{m}]$

Subscripts

Incident sound field

Scattered sound field

Total sound field

Greek Symbols

Total sound field

Imaginary part of the location of a complex monopole source

Spacing on equivalent source surface

Azimuthal angle [degree]

Angular frequency $[\mathrm{rad} / \mathrm{s}]$

Abbreviations

$\begin{array}{ll}\text { BEM } & \text { Boundary Element Method } \\ \text { BPF } & \text { Blade Passing Frequency } \\ \text { BWB } & \text { Blended-Wing Body } \\ \text { CAA } & \text { Computational AeroAcoustics } \\ \text { CESM } & \text { Complex Equivalent Source Method } \\ \text { ESM } & \text { Equivalent Source Method } \\ \text { ESSC } & \text { Equivalent Source Scattering Code } \\ \text { FEM } & \text { Finite Element Method } \\ \text { LEE } & \text { Linearised Euler Equations } \\ \text { SPL } & \text { Sound Pressure Level [dB] } \\ \text { SST } & \text { Source Simulation Technique }\end{array}$

\section{Introduction}

Sound scattering from an airframe is an important feature of aircraft noise. The major sources of aircraft noise

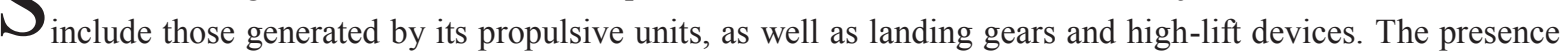
of an airframe could change the incident sound waves and therefore alter the far-field directivity. The scattering effects include reflection, shielding and diffraction by airframe. In order to predict the correct acoustic field surrounding an aircraft, such effects must be taken into account. An accurate and efficient prediction method for the scattering effects could be a useful tool for the design and optimisation of next-generation civil transport aircraft that are able to meet ambitious goals to reduce the impact of civil aviation on the environment ${ }^{1}$. A successful, engineering prediction model of such scattering effect must be able to handle the scattering effect generated by a complex airframe with moderate computational costs. Several analytical and numerical models have been developed for this purpose.

Analytical methods have been developed mainly for scattering effects of a single component, such as a wing or a fuselage. However, most analytical methods can only deal with highly simplified geometries. McAlpine and Kingan $^{2}$ treated the fuselage as a rigid, infinite-long cylinder, while Amiet $^{3}$ modelled a wing as a semi-infinite flat plate. Even though such methods are cheap and easy to implement, they fail to predict the rich sound fields scattered off a complex airframe. A CAA approach either solving Linearized Euler Equations (LEE) or 
employing Finite Element Methods (FEM) to solve the convected wave equations can deal with a complex geometry and flow field. However due to their expensive computational costs and/or deficiencies in the governing equations, they are not yet suitable for full-scale simulations of scattering effects as an engineering tool.

Solving scattering problems with a boundary value technique requires a good compromise between accuracy and cost. In this approach a specific form of the wave equation is solved numerically with appropriate boundary conditions being satisfied on the scattering surfaces. A widely used method is the Boundary Element Method (BEM). It solves the boundary integral equation by discretising the scattering surface into a number of small singularity elements. A linear system is solved to determine the strengths of the elements according to boundary conditions. The elements are then used to construct the scattering field. However, for a traditional BEM, the computation cost is proportional to at least the $2^{\text {nd }}$ power of the number of elements ${ }^{5}$, which places constraints on the size of problem that can be solved. In recent years development of Fast Multiple Method (FMM) partially relieved this problem ${ }^{5}$.

As an alternative to BEM, various forms of Source Simulation Technique (SST) have been developed for analysis of scattering and radiation problems ${ }^{7}$. In such techniques, sources are placed inside the scattering object, underneath the scattering surface, in a way that their acoustic field and incident acoustic field together satisfy the boundary conditions on the scattering surface. With these methods, the number of unknown strengths of sources is far less than the number of control points at which the boundary conditions are satisfied. This leads to an over-determined linear system of smaller scale than that typically solved in BEM. The unknown strengths of the sources are determined by minimising boundary errors. The total acoustic is obtained via super-position of the sound field generated by these sources with the incident sound field. The FMM could also be applied in SST as well ${ }^{19}$.

According to the type of sources the SST techniques can be classified into two categories. The first uses a small numbers of multipoles. Such methods are suitable for either single or multiple scattering objects that have a simple geometry. However, the locations and orders of the multipoles for a complex geometry such as a real airframe are hard to define ${ }^{15}$. Often an optimisation is needed for the two parameters, which increases the workload. The other category places sources of low orders, such as monopoles and dipoles, underneath the scattering surface to form a source surface. Earlier versions are under the name of 'wave superposition approach $^{8}{ }^{9}{ }^{10}$. In recent years, Fast Scattering Codes (FSC) for large-scale aeroacoustic scattering problems were developed under the name of 'equivalent source method' (ESM) in the frequency domain ${ }^{11}{ }^{12}$. A timedomain version of this method is developed and tested by Lee et al. ${ }^{13}{ }^{14}$. Gounot et al. summarised some of the approaches and outlined guidelines for application of equivalent source method ${ }^{15}$.

Ochmann $^{7}$ reviewed the source simulation approaches under different names. He pointed that out that compared to BEMs, the SSTs including ESM could reduce BEM's intense computational costs that arise from treating complex structures by involving a large numbers of elements. Meanwhile the SSTs including ESM could avoid singular integrals. SSTs have disadvantages, for example, their usage is not as automatic as BEM since an additional source surface must be generated other than the scattering surface. However such minor disadvantages would not adversely affect its potential in simulation of full-scale real aircraft.

In section II of this paper, the traditional ESM is introduced briefly with discussions of its disadvantages. Then a new complex ESM (CESM) will be developed based on complex monopoles. Validation of the new method is discussed in Section III and Section IV using 2D and 3D cases respectively. Section VI summaries the current work and proposed future works.

\section{Complex Equivalent Source Method (CESM)}

\section{II.A Traditional Equivalent Source Method}

A homogeneous, convective wave equation with a uniform flow in frequency domain can be written as 
where $P$ is the acoustic pressure, $k$ is the wavenumber and

$\mathbf{U} / c_{0}$ is the free stream Mach number vector. The free space, moving medium, frequency-dependent Green's function given by Blokhintsev ${ }^{16}$ is

$$
G\left(\begin{array}{ll}
\mathbf{x} & \omega
\end{array}\right)-
$$

where $S$ and $r_{e}$ is the amplitude and phase radius defined as

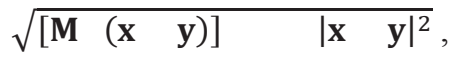

$$
\begin{aligned}
& -\left[\begin{array}{lll}
- & (\mathbf{x} & \mathbf{y}
\end{array}\right)
\end{aligned}
$$

where $\quad|\mathbf{M}|^{2}, \mathbf{x}$ and $\mathbf{y}$ is the visual location of observer and source. With this function, the pressure by monopole of strength can be written as

$$
\left(\begin{array}{lll}
\mathbf{x} & \omega
\end{array}\right) \quad(\mathbf{y} \omega) G\left(\begin{array}{ll}
\mathbf{x} & \omega
\end{array}\right) .
$$

This basic solution is the essential brick for establishing an ESM computational model. Figure 1 shows a schematic of the principle of equivalent source method. A total of $N_{x}$ control points (red points) are distributed on the scattering surface, and a total of $N_{y}$ equivalent monopole sources (blue points) are distributed interior to the scattering surface. At each control point, the scattering pressure field is the super-position of the sound field generated by each equivalent monopole. This yields a linear system

$$
[A \quad,
$$

whose elements are

$$
G\left(\mathbf{x}_{\mathbf{i}}, \mathbf{y}_{\mathbf{j}}, \omega\right)
$$

Similarly the normal gradient of the pressure at all control points can be written in a linear system

$$
[B] Q_{e q},
$$

whose elements are

$$
G(\mathbf{x} \quad \omega) \quad \nabla G(\mathbf{x} \omega)
$$

Since the locations of control points and equivalent sources are determined, and could be evaluated explicitly from expression of Green's function $G(\mathbf{x}, \mathbf{y}, \omega)$. 


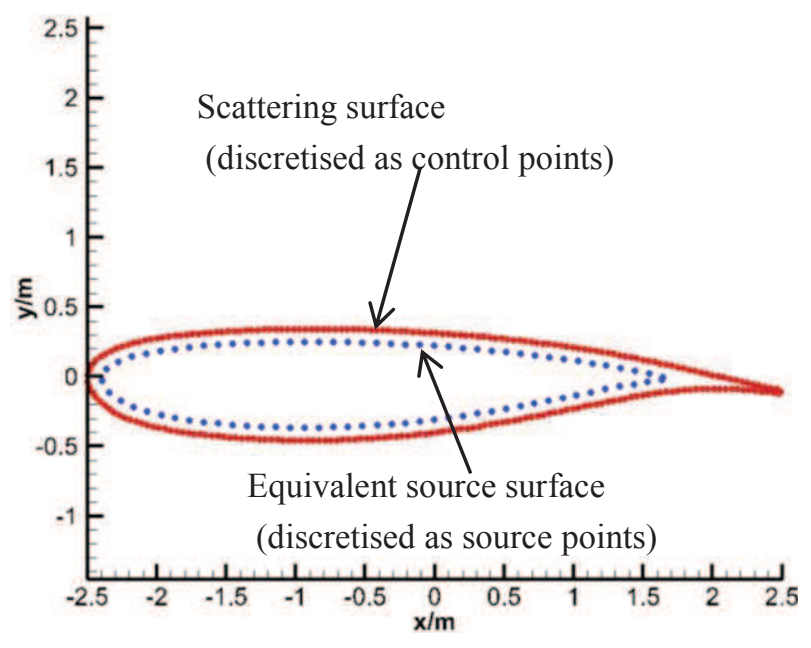

Figure 1. Schematic of traditional ESM.

On the scattering surface, the impedance boundary conditions are satisfied, which can be written as

where $P$

is the total acoustic pressure obtained by superposition of incident pressure

and scattering pressure and $Z$ is the acoustic impedance. Therefore,

$$
(P \quad)+\square \nabla_{n}\left(P_{i n}+P_{s c}\right)=0 .
$$

By substituting expression for and that the following expression can be obtained

$$
\left(\begin{array}{lll}
{[A} & -[B]
\end{array}\right) Q \quad-\left(\begin{array}{ll}
P & -
\end{array}\right) .
$$

If the incident pressure field is known, then the right-hand side of this system can be calculated. This system is a complex, over-determined linear system of size $N_{x} \times N_{y}$ which can be solved by a least squares methods. The solution for $Q_{e q}$ is then used to construct the total exterior pressure field using
$(\mathbf{x})$
$(\mathbf{x})$
$(\mathbf{x})$
(x) $\quad \sum$
$(\mathbf{y} \omega) G(\mathbf{x}$
$\omega)$.

Although the methodology of ESM is straight-forward, its numerical implementation is not trivial. The main difficulty lies in the construction of equivalent monopole sets. No general rules or guidelines exist, and the generation of scattering surface is done in an ad hoc manner. Most previous work suggest that the source surface should be a similar shape to the scattering surface, but smaller in scale and placed interior to the scattering surface. Tinetti and Dunn ${ }^{12}$ suggested that the source surface should be curved to ensure a constant distance from the scattering surface. However, the choice of the distance is ambiguous. If the source surface is placed too close to the scattering surface, the calculated strengths of equivalent monopoles must be small enough to satisfy the boundary conditions on the scattering surface. However, they can be too small to construct the scattering field in the far-field. If the distance is too large, the resulting small source surface is very likely to lead to a badly conditioned linear system. Furthermore, for a scattering object which has thin, sharp edges, such an appropriate universal distance may not exist. For example, an appropriate distance for the airfoil leading edge tends to be large for the thin, sharp trailing edge. As shown in Figure 1, near the trailing edge the distance from control points to the source surface is much larger than that near the leading edge. This could lead to large 
boundary errors near the trailing edge, thus the scattering effects, especially diffraction and shielding by the thin sharp trailing edge, could be lost. Figure 2 highlights the problem, showing the scattering of sound field of a 2D line source placed at $(0.0,-5.0)$ by a RA16SC1 arifoil, using traditional ESM and the computational model in Figure 1.

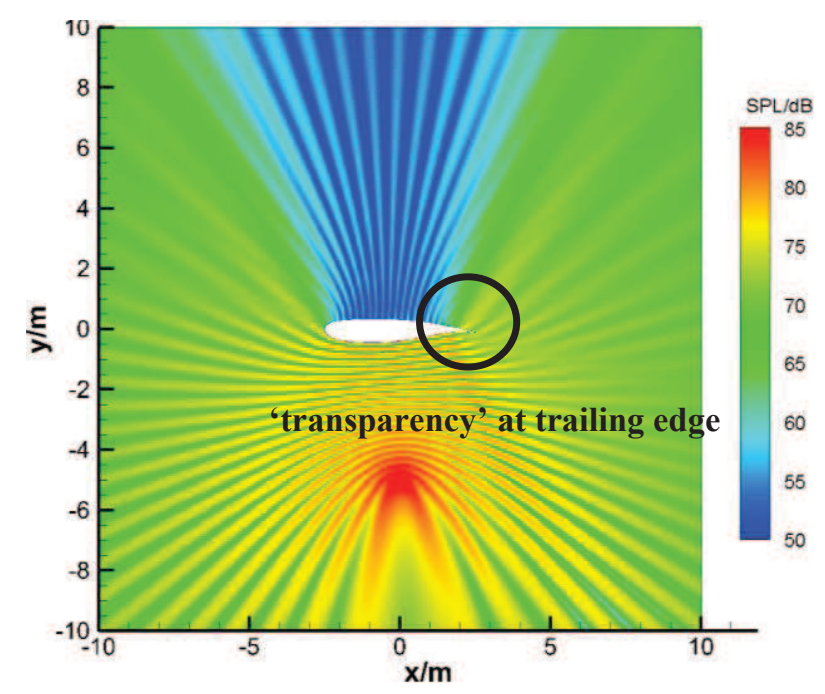

Figure 2. Scattering of a line source off a 2D airfoil, predicted by traditional ESM.

In Figure 2, it can be seen that the airfoil trailing edge seems to be 'transparent' to the incident sound wave. A diffraction lobe goes through the thin, sharp trailing edge. This disadvantage was also addressed by Tinetti and Dunn ${ }^{12}$. They suggested that flat plates should be used to replace thin scattering objects that have thin, sharp edges. However, such a treatment is counter to the purpose of numerical methods to treat real, complex geometries. For a scattering body that has both bluff bodies and thin edges this treatment is not practical. New approaches are needed.

\section{II.B Complex Equivalent Source Method}

We now develop a Complex Equivalent Source Method (CESM) for aircraft noise problems, which improves upon the traditional ESM. In CESM, the positions of equivalent monopoles are in complex space instead of real space ${ }^{18}$. Such a complex source has coordinates defined as

$$
\left(\begin{array}{ll}
a & c
\end{array}\right)
$$

where $i=\sqrt{-1}$. Such a monopole is also a basic solution with respect to the real spatial coordinates $\boldsymbol{x}$ $\left(x_{1}, x_{2}, x_{3}\right)$ for a fixed complex source position $\boldsymbol{y}_{\boldsymbol{c}}$. The proof can be found in Ochmann ${ }^{17}$ for a static medium, and it's easy to extend to a moving medium. Such a monopole behaves in a completely different way as common real sources. It is singular on a disc instead of a single point, and the sound is mainly radiated in a half space and concentrated in the paraxial region near the axis given by $\boldsymbol{\gamma}$, when the magnitude of $\boldsymbol{\gamma}$ or the wavenumber $k$ is not too small. Figure 3 is an example of a such point source with $\mathbf{y}=(0,0,0)$ and $\boldsymbol{\gamma}$ $|\boldsymbol{\gamma}|\left(\frac{\sqrt{2}}{\sqrt{ }}, \frac{\sqrt{ }}{0}\right)$. From the figure it can be seen that as the magnitude of $\boldsymbol{\gamma}$ increases, the sound field becomes concentrated and the source disc becomes larger. Applications of such sources have been performed by Ochmann and Piscoya ${ }^{18}$ to a sound radiation problem with promising accuracy. 


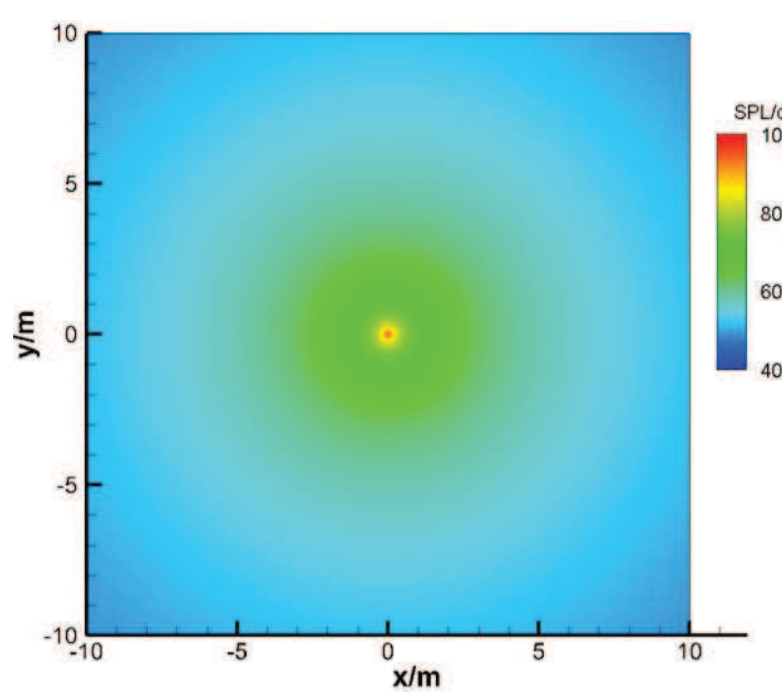

(a) $|\gamma| \quad 0$.

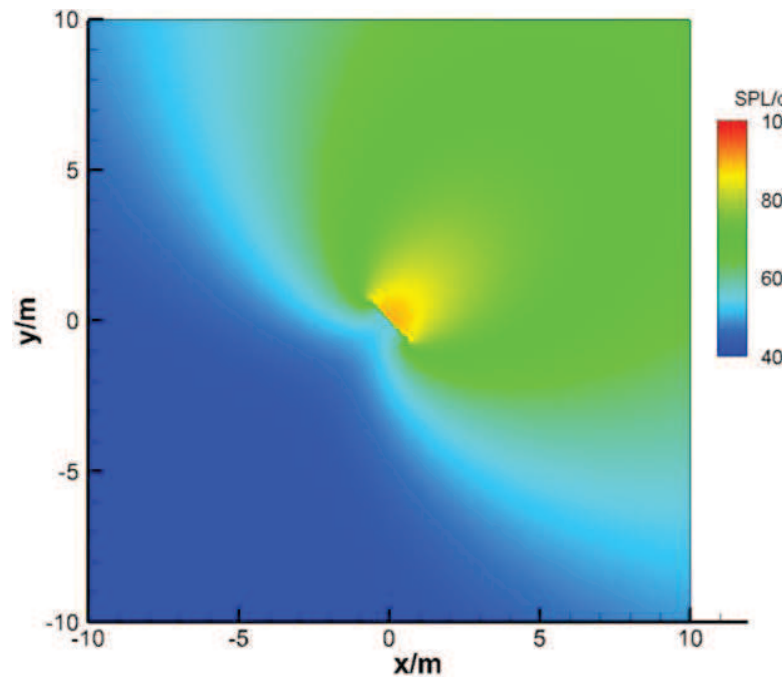

(c) $|\gamma|$

0.

Figure 3. Sound from a complex monopole located at

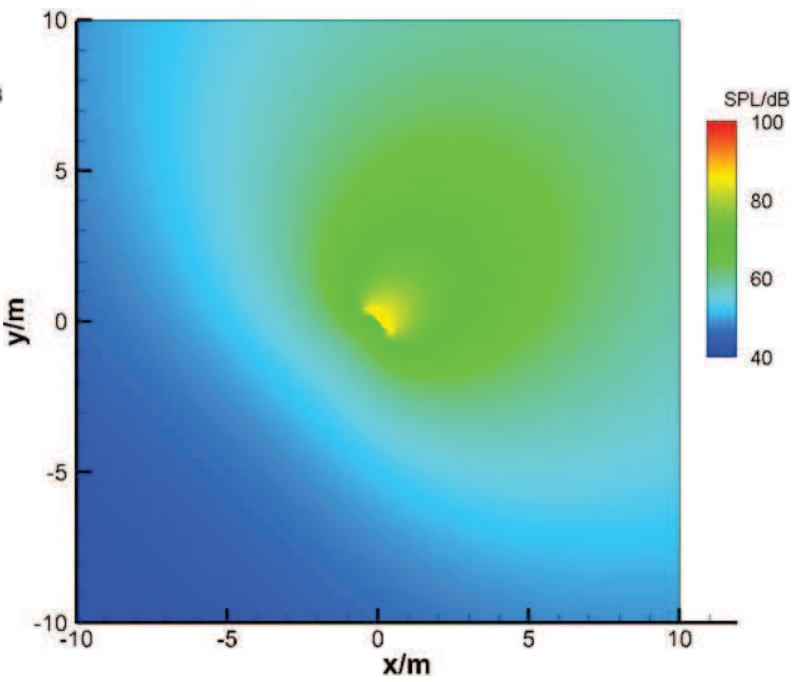

(b) $|\gamma|$

5.

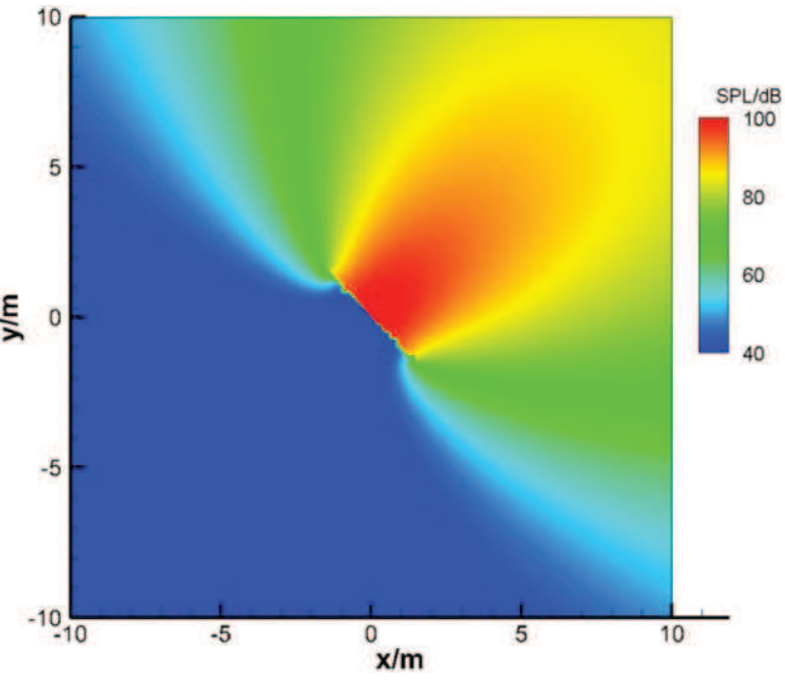

(d) $|\gamma|$

0.

From Figure 3 it can be seen that, unlike a real monopole, the complex monopole has much smaller gradient of sound pressure amplitude close to its projection in real space. This allows the projection of equivalent complex monopoles in real space to be placed close to the scattering surface. Thus thin bodies and sharp edges could be treated with improved accuracy.

Like normal ESM, the scattering surface construction has no general rules. However, it is reasonable to place the complex monopole source disc interior to the scattering surface, so that the singularity disc would not affect the exterior sound field. The basic idea is to move the scattering surface inside in the normal direction of scattering surface defined by $\mathbf{n}_{\mathbf{x}}$,

where is the distance defined while a real scaling factor . The distance is defined via

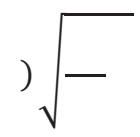


where $V$ is the volume of the scattering object. In order to ensure all complex monopoles to radiate sound outside, the normal vectors of the source surface (pointing outside) are used for $\boldsymbol{\gamma}$. An imaginary scaling factor $s c a l e_{i}$, and the spacing of monopoles on scattering surface $\Delta_{s}$ are used to control the magnitude of $\boldsymbol{\gamma}$.

$$
-i\left(\text { scale }_{i} \Delta_{s} \mathbf{n}_{\mathbf{y}}\right)
$$

If the complex factor is set as 0 , then the traditional ESM with real monopoles is recovered.

\section{Verification using 2D Test Cases}

To verify the new CESM method, simulations are performed on benchmark test cases test. In a 2D space, the free field Green's function in a static medium can be written as

$$
{ }_{0}^{(2)}(k|\quad \mathbf{y}|)
$$

where $H_{0}^{(2)}(z)$ is the second-kind Henkel function of zero order. Three different cases are studied with results presented in the following sections III.A to III.C.

\section{III.A Scattering of a line source off a circular cylinder}

An analytical solution for scattering of a line source off a circular cylinder exists; this case is used for validation of CESM methodology. The analytical solutions can be found in $\mathrm{Mechel}^{4}$. In this case the scattering object is a rigid circular cylinder centred at the origin with a radius of 2.5 metres. The incident sound field is generated from a unit line source placed 5.0 metres below. The observers are placed on a ring $4 \mathrm{~m}$ from the origin. Three frequencies, $200 \mathrm{~Hz}, 800 \mathrm{~Hz}$ and $1600 \mathrm{~Hz}$ are simulated. On the scattering surface, a total of 20 points per wavelength are used to resolve the scattering surface for the $200 \mathrm{~Hz}$ case and 10 points per wavelength are applied for the two higher frequencies. The number of equivalent monopoles is chosen to be $1 /$ of the number of control points, following Tinetti and Dunn ${ }^{12}$ for traditional ESM. A code named Equivalent Source Scattering Code (ESSC), which applies CESM and written in MATLAB, is used for the three case studies.

Validation of results is shown in Figure 4. On the observer ring the shielding effects are obvious for which indicates the boundary of the shadow zone created by the cylinder. Within this region the total sound deceases towards the farthest point from the source at $\phi=270^{\circ}$. As the frequency increases the effectiveness of attenuation improves and the oscillation increase. For all three frequencies, the CESM predictions achieve good agreement with analytical solutions. This means that for thick bodies CESM has the same capability as the traditional ESM. 


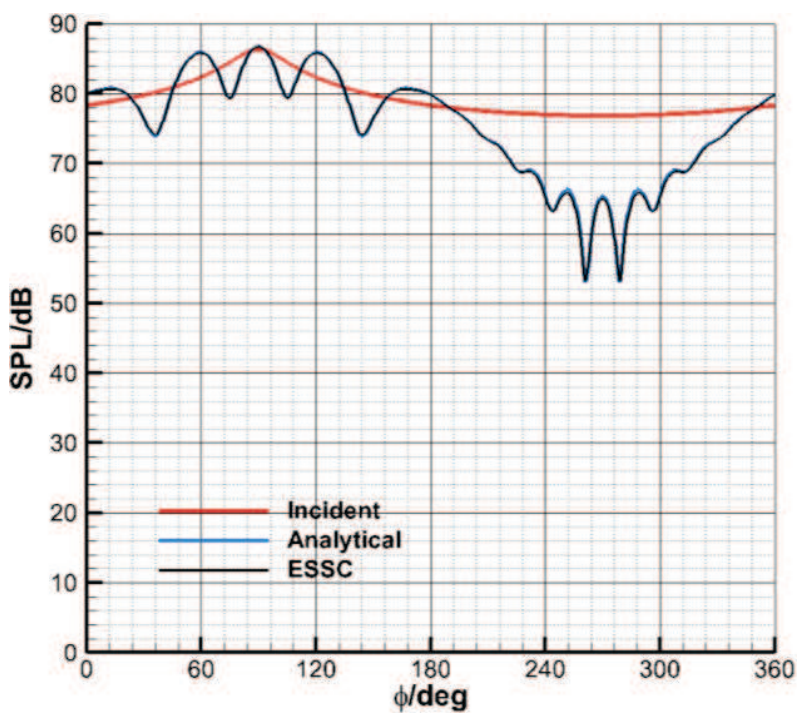

(a)

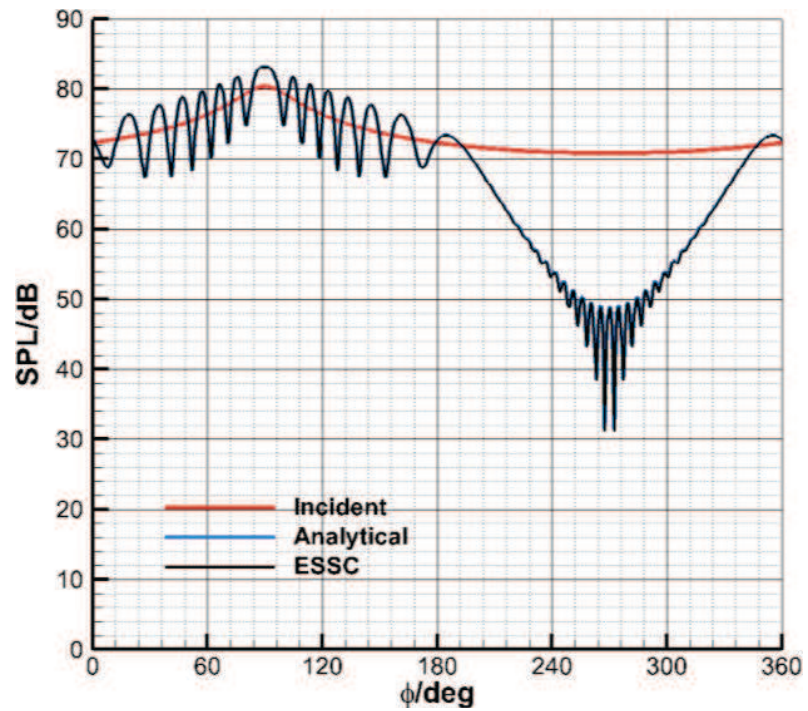

(b)

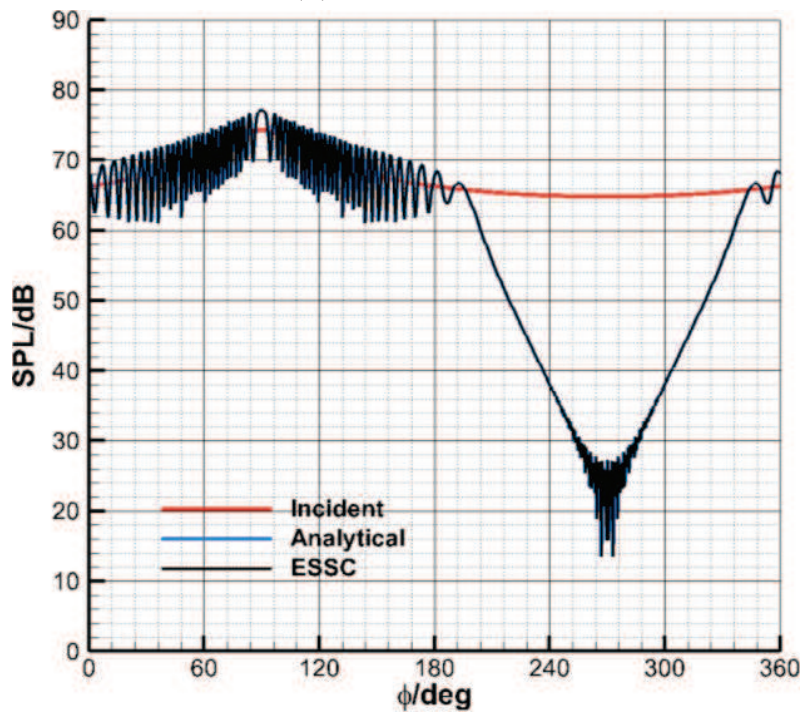

(c) $f=3200 \mathrm{~Hz}$.

Figure 4. Scattering of sound field of a line source off a circular cylinder. 


\section{III.B Scattering of a line source off $2 \mathrm{D}$ bodies with elliptic and rectangular cross-sections}

Test cases of 2D bodies with elliptical and rectangular cross-sections are also used in this study. These cases are designed to test the CESM's capability of treating thin scattering objects. Since no analytical solutions exist for these cases, the prediction results can only be analysed qualitatively. The scattering object is a $2 \mathrm{D}$ column with either a rigid elliptic cross-section or a rectangular cross-section, centred at the origin with a width of 5.0 metres. The aspect ratios are 1.0, 0.1, 0.01 and 0.005 respectively. Incident sound field is generated from a unit line source placed 5.0 metres below. One frequency of $800 \mathrm{~Hz}$ is simulated. 10 points per wavelength are used to resolve the scattering surface. The number of equivalent monopoles is again chosen to be $1 / 3$ of the number of control points. The resulting sound pressure level (SPL) contours are shown in Figures 5 and 6. From the results it can be seen that a well-defined shielding shadow zone can be found for all simulations behind the scattering object, which illustrates that the shielding effect is successfully captured. Meanwhile obvious regions of amplification due to reflection below the scattering bodies are well predicted. The diffraction effect near the edges, which is indicated by lobes bending towards the shadow zone, is well captured by CESM, even for very thin scattering bodies with sharp edges.

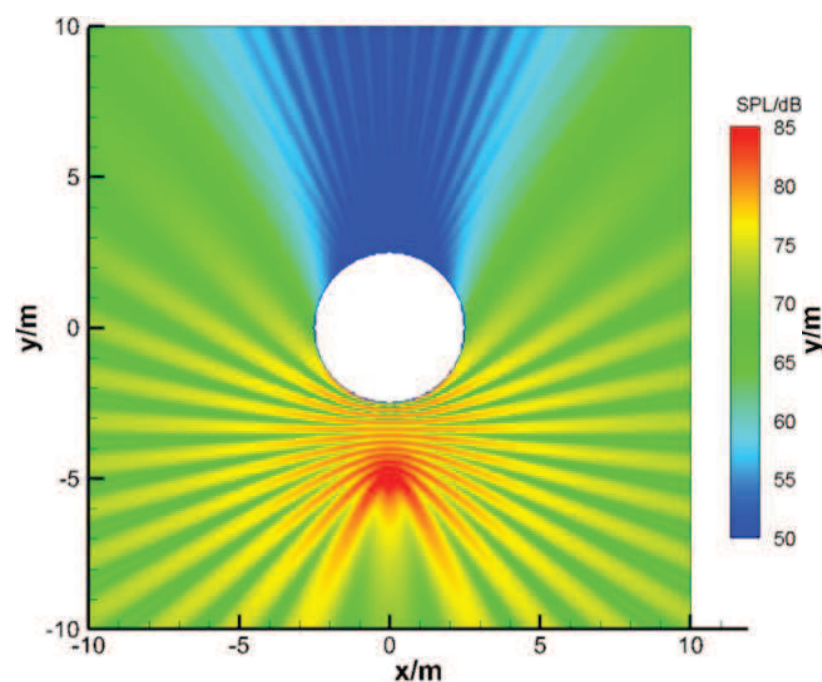

(a)

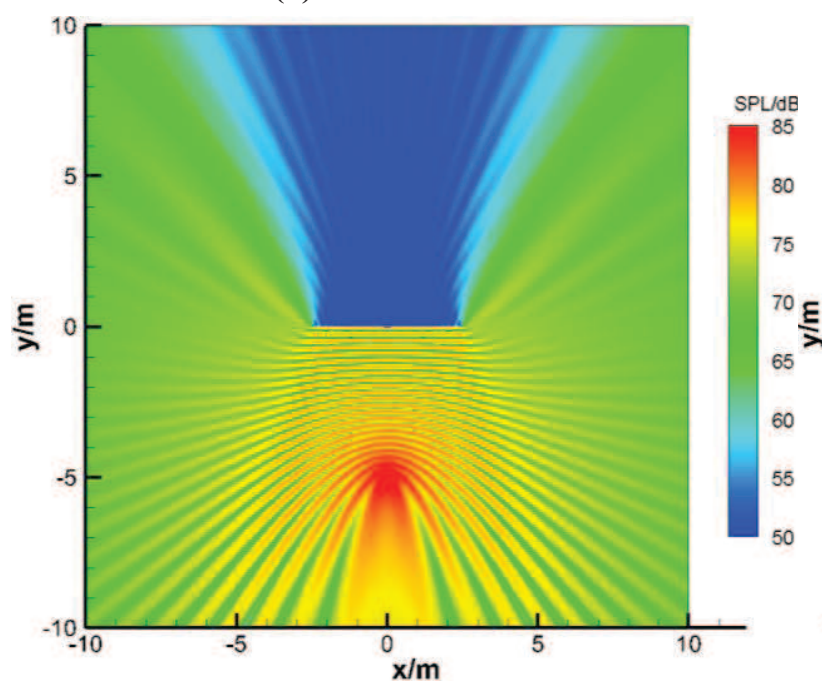

(c)

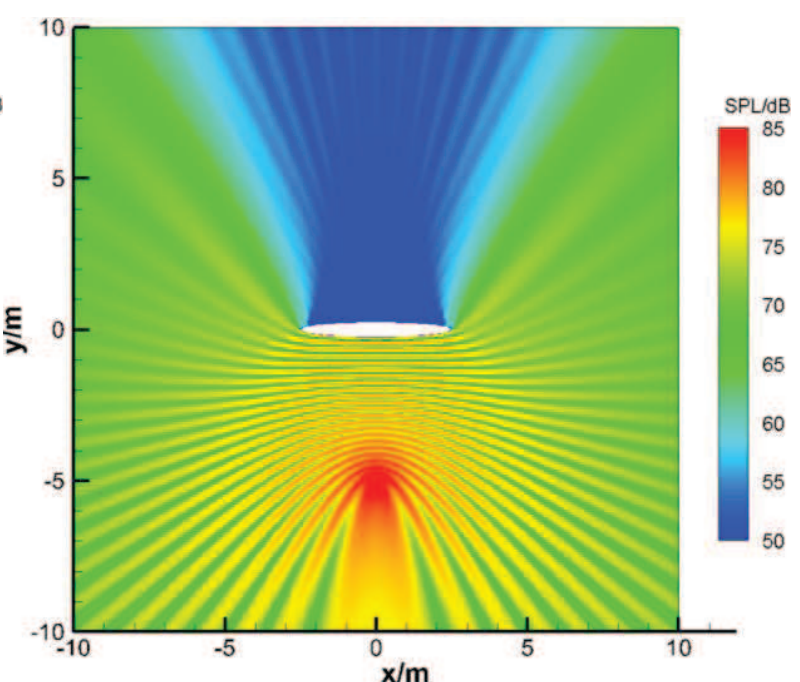

(b)

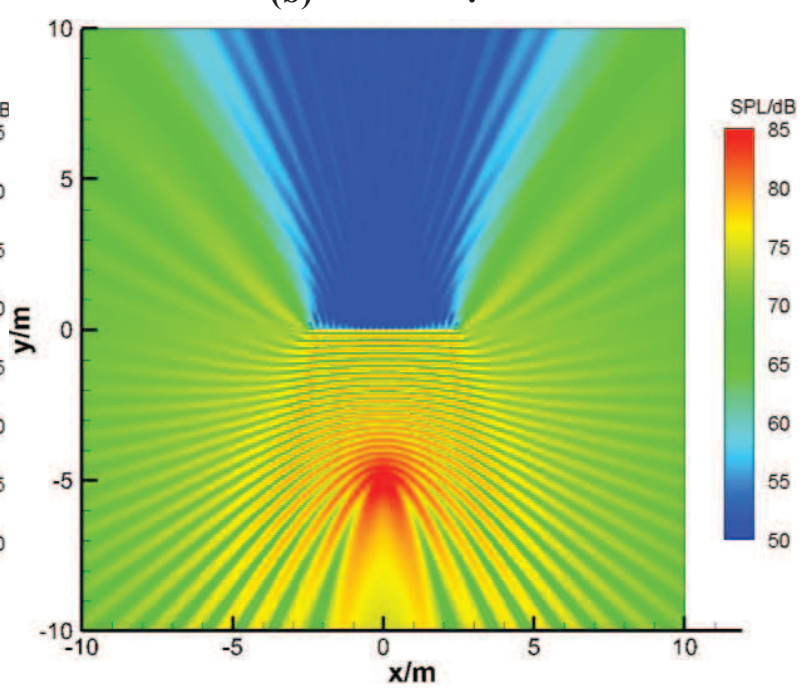

(d)

005.

Figure 5. Sound from a line source and scattered off an elliptic cylinder. 


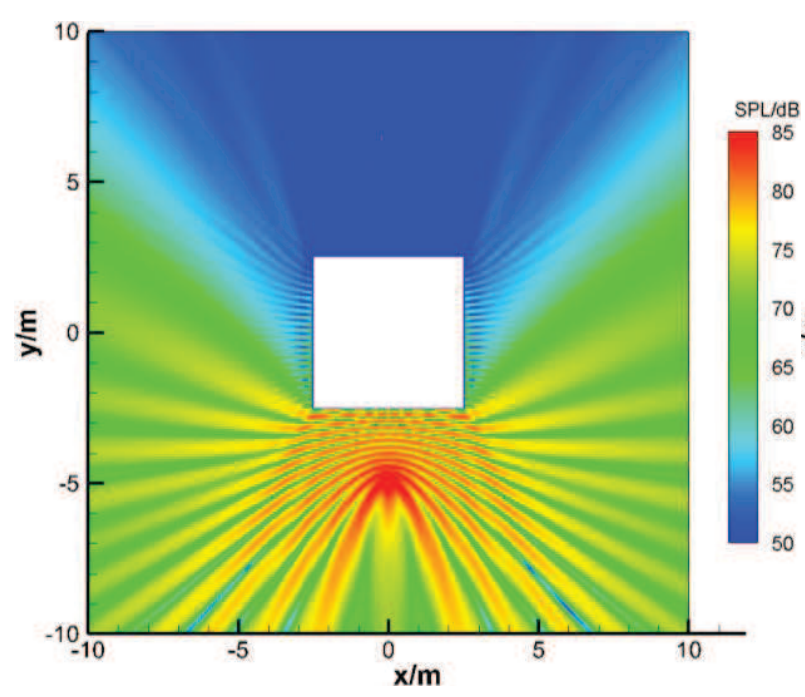

(a)

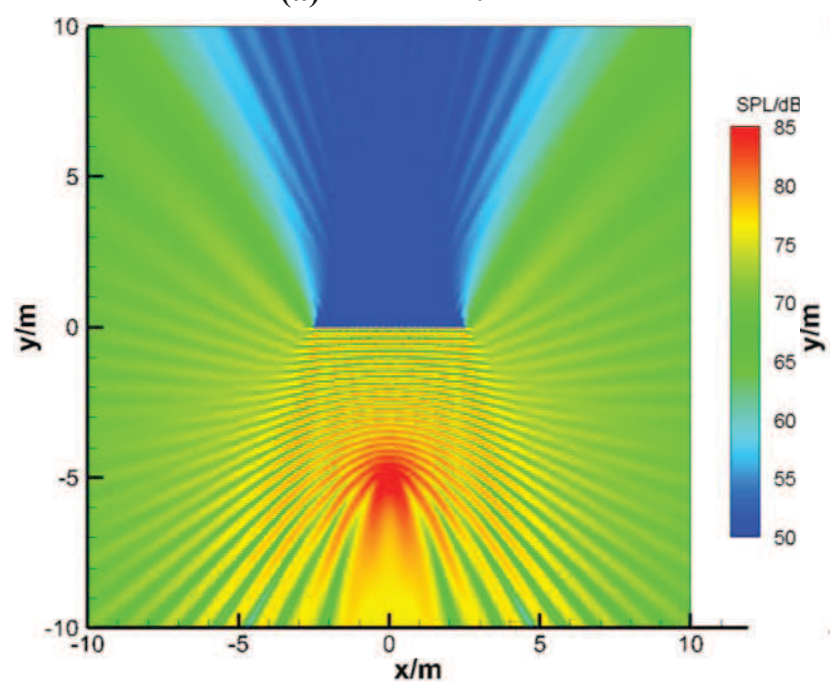

(c)

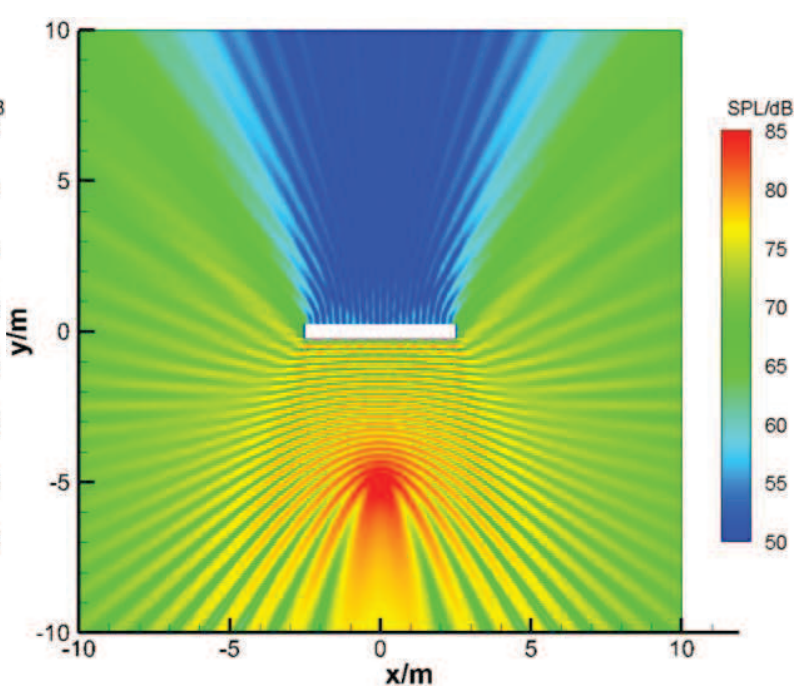

(b)

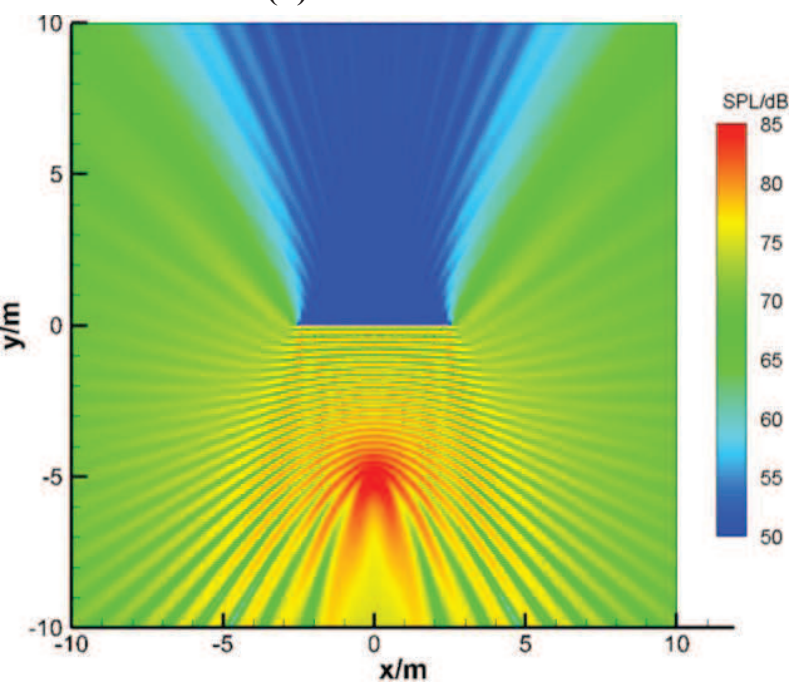

(d)

005.

Figure 6. Sound from a line source and scattered off a rectangular cylinder.

\section{III.C Scattering of a line source off a 2D airfoil}

These simulations aim to testing CESM's capability in simulating airfoil-like scattering objects. The scattering object is a 2D RA16SC1 airfoil with a chord length of 5 metres. The incident sound field is generated from a unit line source placed 5.0 metres below the airfoil. One frequency of $800 \mathrm{~Hz}$ is simulated. On the scattering surface, a resolution of 10 points per wavelength is applied. The number of equivalent monopoles is chosen to be $1 / 3$ of the number of control points. The computational models and predicted SPL contours for traditional ESM and CESM are shown in Figures 7 and 8 respectively. 


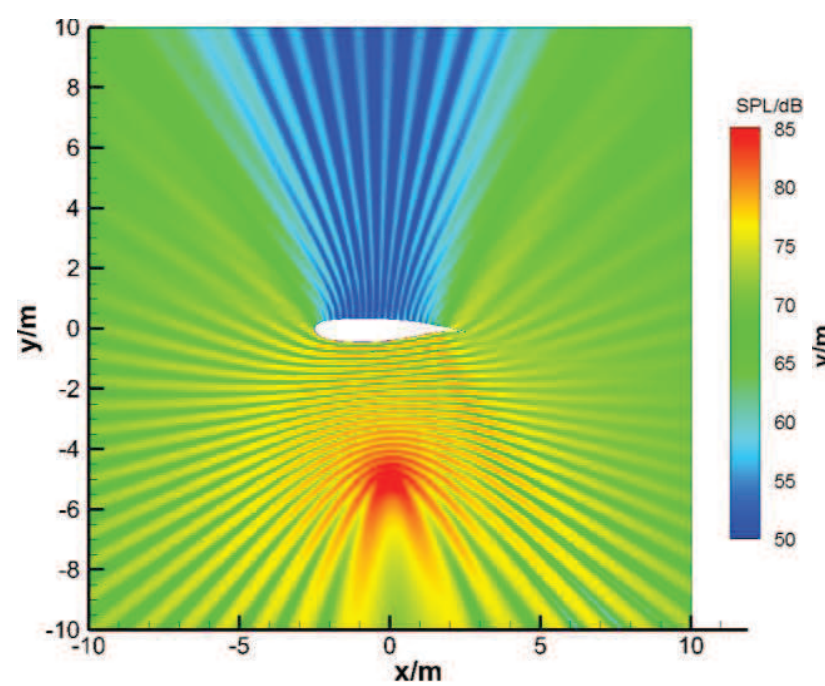

(a) Total acoustic field.

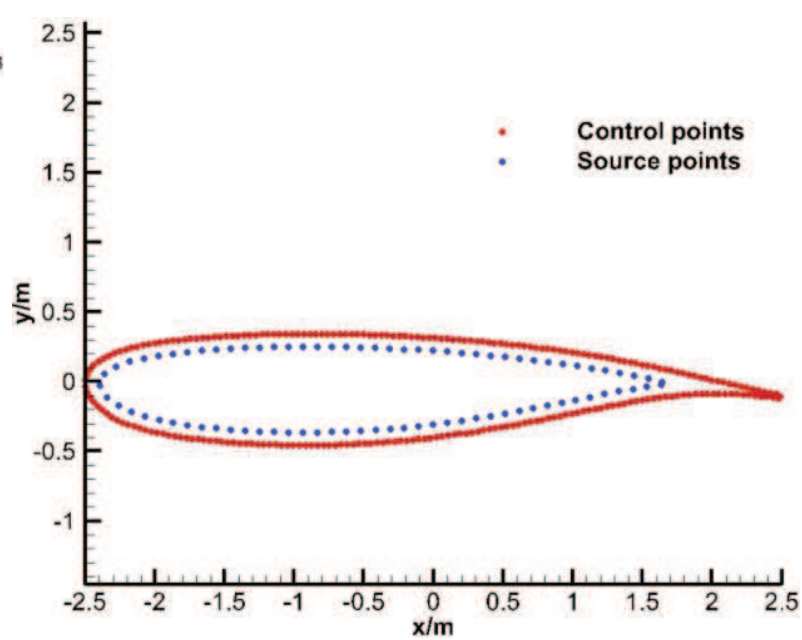

(b) Computational model.

Figure 7. Sound from a line source and scattered off a 2D airfoil predicted by traditional ESM.

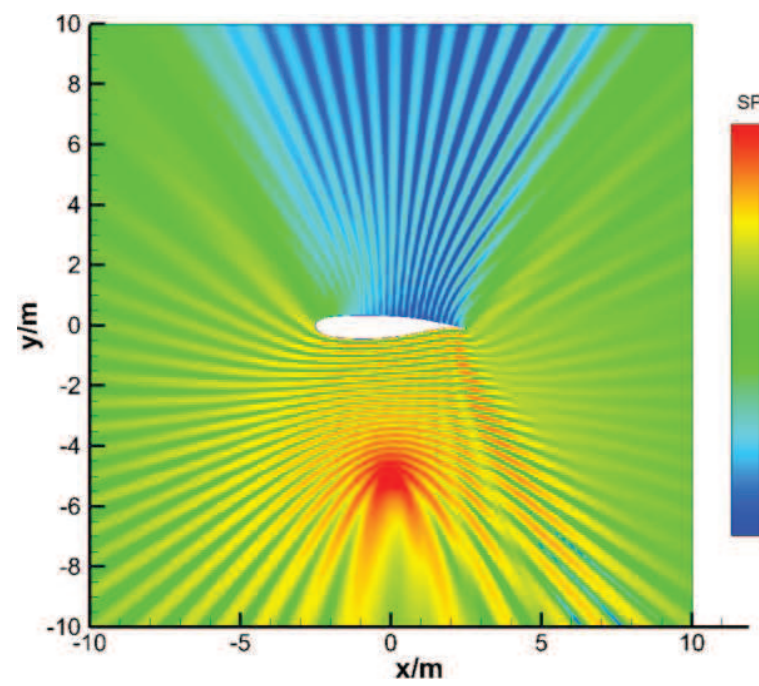

(a) Total acoustic field.

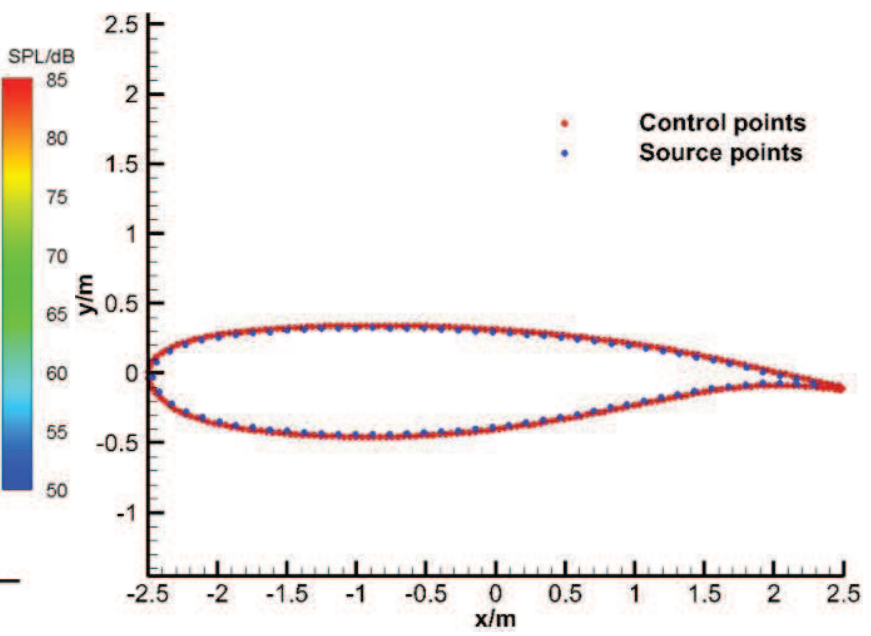

(b) Computational model.

Figure 8. Sound from a line source and scattered off a 2D airfoil predicted by CESM.

It can be seen that the CESM allows the source surface to be placed very close to the scattering surface, which is not possible for the traditional ESM. The traditional ESM predictions are not satisfactory. It is noted that even though some diffraction patterns are captured, the trailing edge seems to be 'transparent' to the incident waves, as one diffraction lobe is obviously captured penetrating the trailing edge. Meanwhile, the diffraction/interference patterns are more symmetric, which means the difference of smooth leading edge and sharp trailing edge is not effectively distinguished by the ESM prediction. In contrast, in the CESM prediction no obvious lobes penetrate the trailing edge of the airfoil, and a region of enhanced reflection patterns is captured below the highly-cambered trailing edge bent towards the source. This suggests that CESM can overcome the disadvantages of the traditional ESM in treating the scattering effect at a sharp edge. 


\section{3D Scattering off a Rigid Cylinder}

In this section simulation results of a 3D benchmark case are presented, for the purpose of testing the capability of CESM in a 3D space. The scattering effect of a ring of rotating monopole/dipole sources by a rigid cylinder is simulated and validated against the analytical solutions by McAlpine and Kingan ${ }^{2}$. As shown in Figure 9, this case is a highly simplified representation of a scattering problem consisting of a rotor and a fuselage. The rotating monopoles/dipoles are the simplest representations of rotor thickness/loading noise, and the scattering body is a fuselage simplified as a cylinder. Six steady unit monopoles/dipoles are distributed evenly on a ring which has a radius of 1.0 metres. The ring's rotating axis is parallel to that of the scattering circular cylinder. The ring is placed 4.0 metres away from the cylinder axis on the $y$ axis, which corresponds to an azimuthal angle of $0^{\circ}$. The scattering circular cylinder, on whose surface a rigid acoustic boundary condition is applied, has a radius of 2.0 metres and a finite length of 20 metres. Since the analytical solution is valid for far-field noise, the observers are located far from the cylinder on the plane of rotating sources, 100 metres away from the cylinder axis. The non-dimensional rotation speed of sources $M_{t}$ is 0.5 , and the free-stream has a Mach number of 0.7 and is parallel to the cylinder's axis. In total three simulations are performed with acoustic modes

,3 which correspond to frequencies of 164,328 and $491 \mathrm{~Hz}$ respectively. A total of 18608 control points are distributed on the scattering surface. The sources surface's diameter is 0.95 times the diameter of the rigid cylinder, on which a total of 6336 complex monopoles are placed.

The simulations are performed on a workstation with 8 processors and 16 GB RAM with the ESSC code. Each simulation took less than 200 seconds. Results are shown with analytical solutions in Figures 10, 11 and 12 .

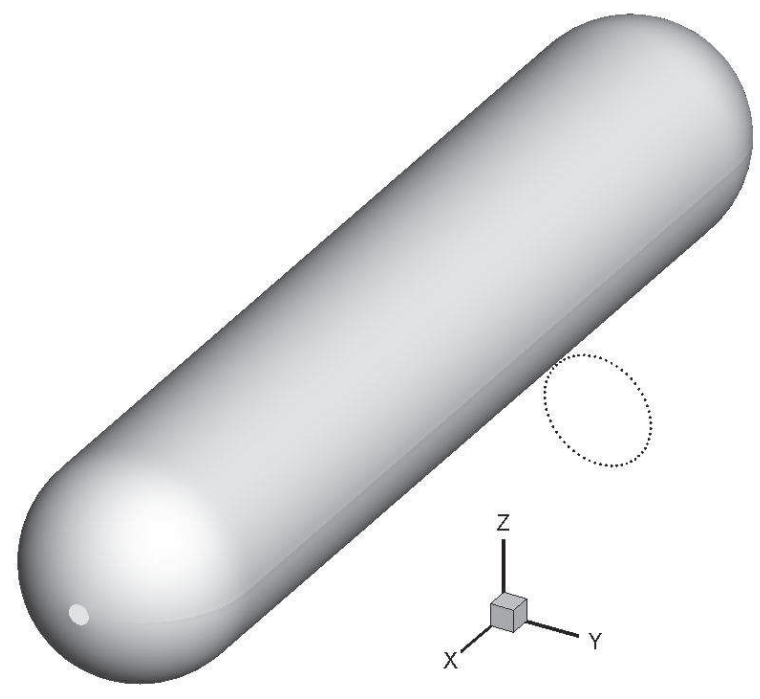

(a) Geometry of simulations.
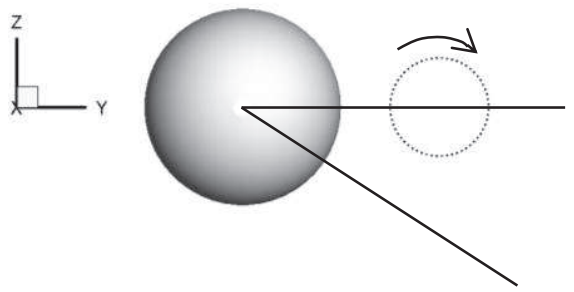

(b) Definition of azimuthal angle $\phi$.

Figure 9. Schematic of rotating sources and 3D scattering cylinder body. 


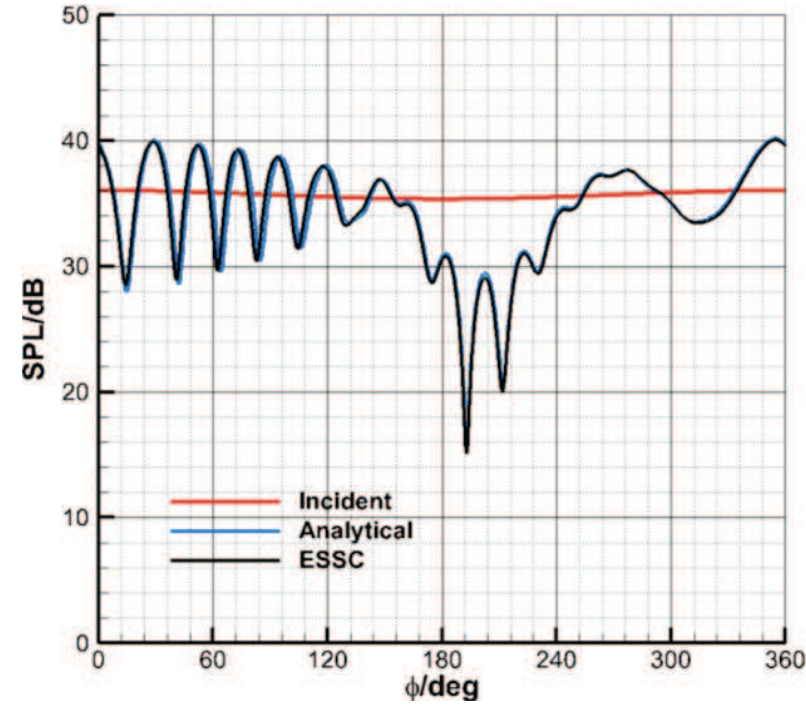

(a) Results of rotating monopoles.

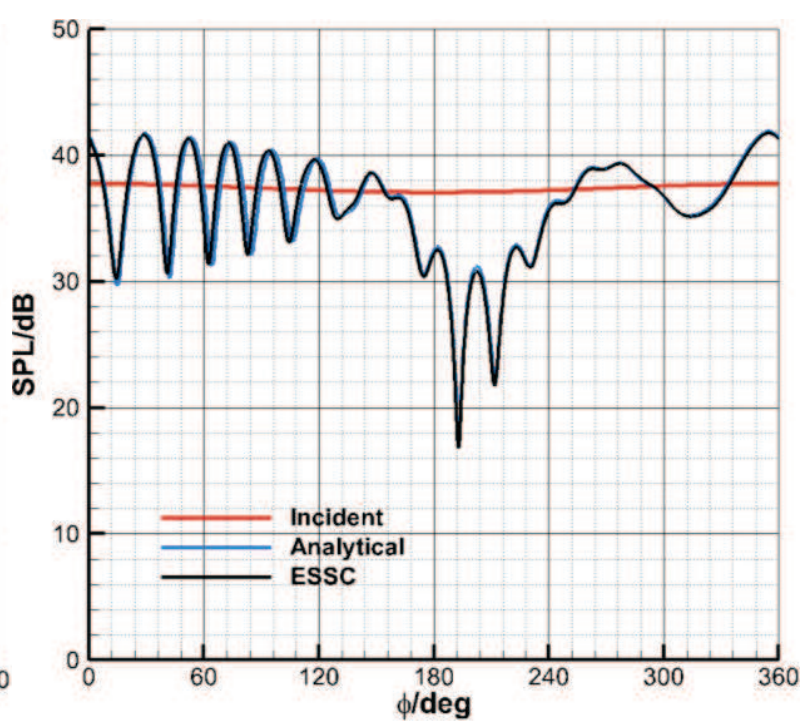

(b) Results of rotating dipoles.

Figure 10. Sound from rotating monopoles/dipoles scattered off a finite cylinder,

Hz.

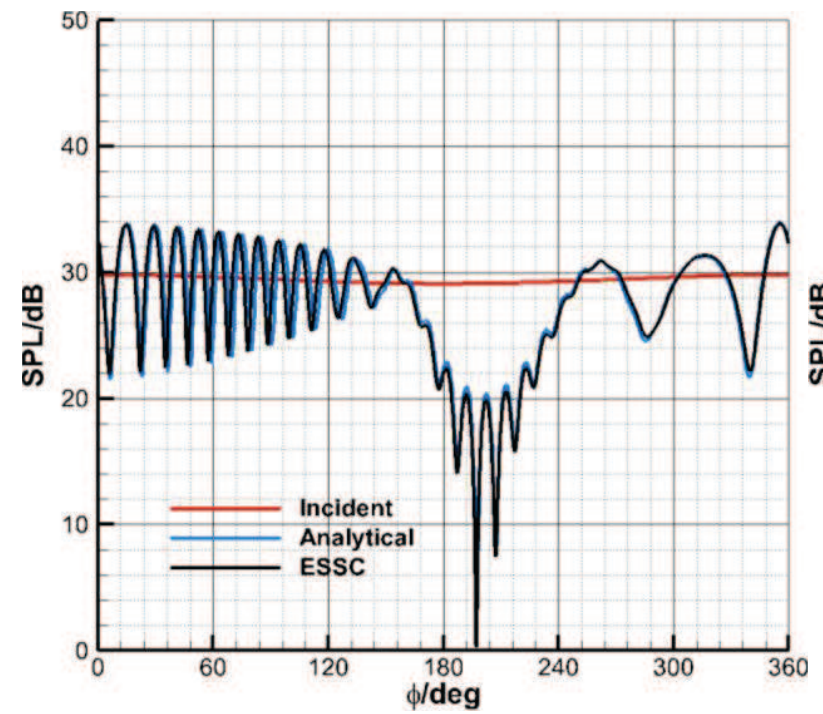

(a) Results of rotating monopoles.

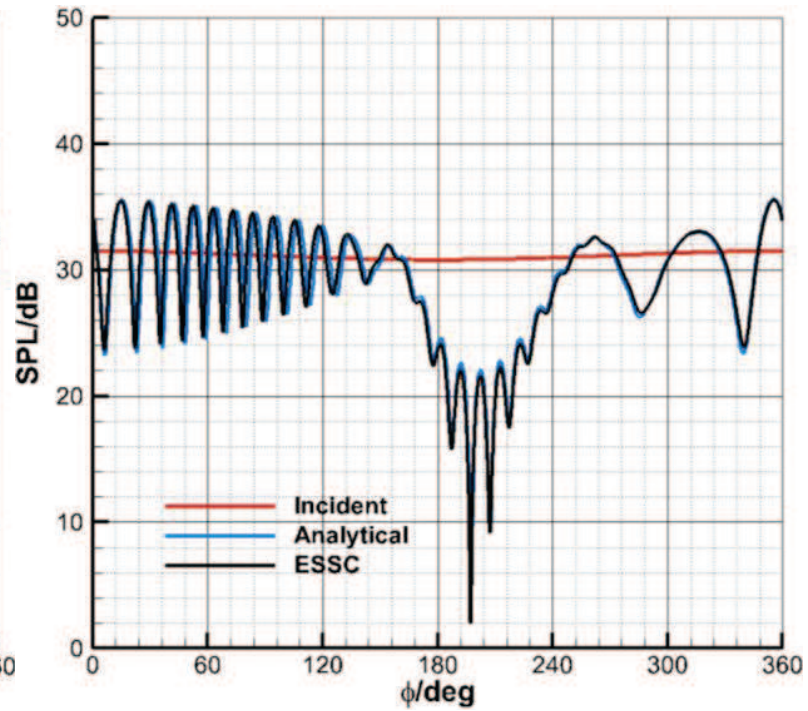

(b) Results of rotating dipoles.

Figure 11. Sound from rotating monopoles/dipoles scattered off a finite cylinder,

Hz. 


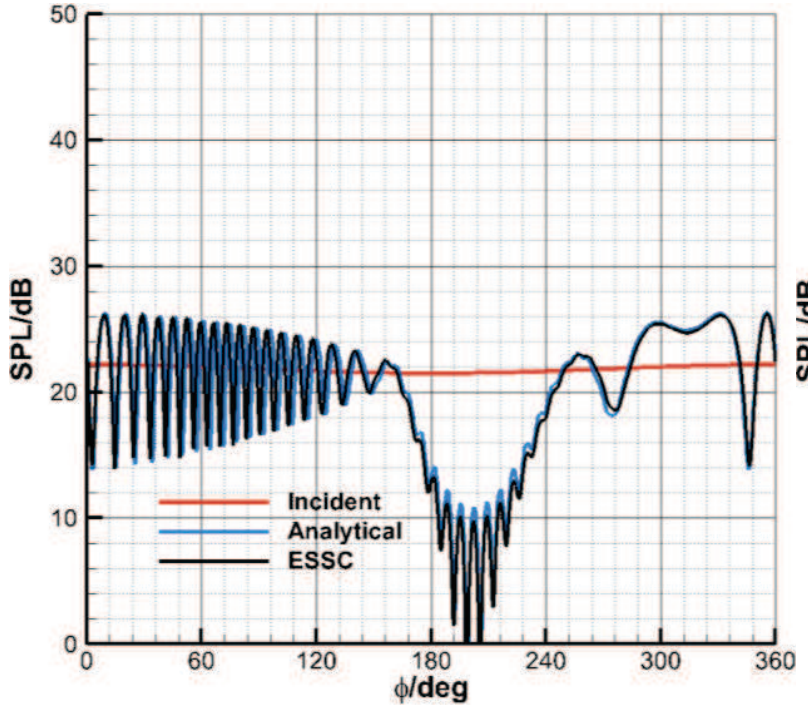

(a) Results of rotating monopoles.

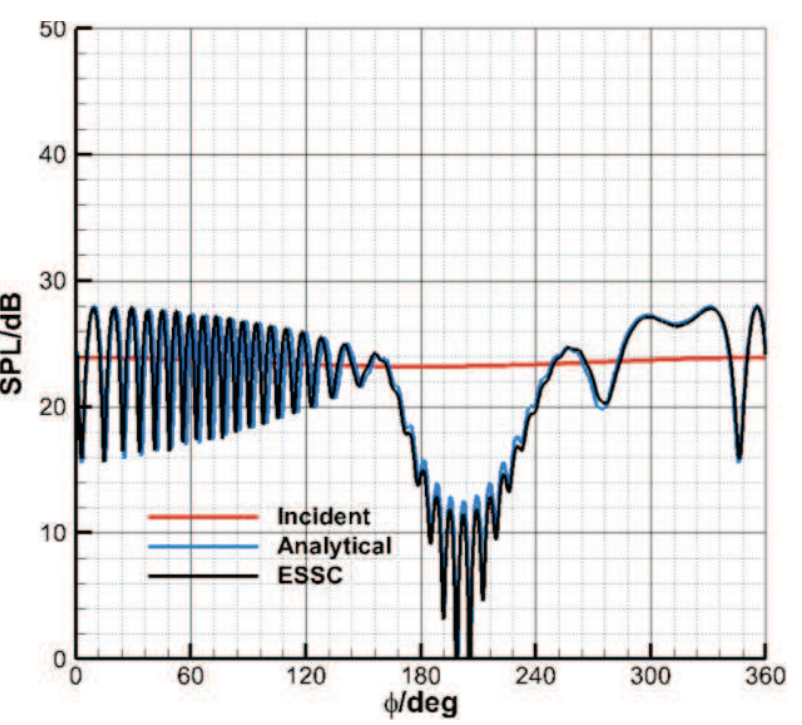

(b) Results of rotating dipoles.

Figure 12. Sound from rotating monopoles/dipoles scattered off a finite cylinder,

A well-defined shielding region can be observed at all three frequencies. However, the shadow zone is not centred around $\phi=180^{\circ}$, but biased within the region $160^{\circ}<\phi<250^{\circ}$. Further examination of the system shows that the sources are mostly shielded when the direction of their rotating velocity vectors are pointing towards the cylinder surface. The maximum attenuation of sound occurs around $\phi=200^{\circ}$ where the directivity patterns are highly oscillatory. Similar to the $2 \mathrm{D}$ cylinder cases, the attenuation improves as the frequency increases as a result of shortening wavelength. As expected, more lobes appear with increased frequency, which indicates a higher level of oscillation.

The directivity patterns calculated by CESM agree well with the analytical solutions of McAlpine and Kingan $^{2}$. Generally the directivity patterns obtained by CESM are coincident with analytical solutions, except around $\phi=90^{\circ}$ where the numerical results are biased very slightly from the analytical results. The differences between the analytical solution and CESM results are smaller than $1 \mathrm{~dB}$ for all azimuthal angles on the observer ring at all three frequencies.

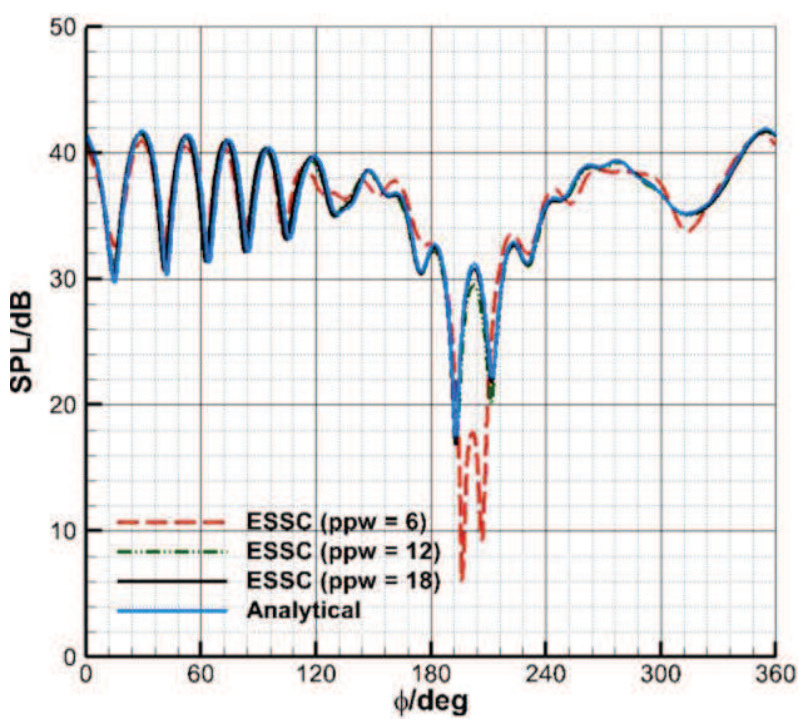

Figure 13. Effect of resolution on scattering surface for 
From the figures it can be noticed that the largest discrepancy occurs at $m=3$. Recall that the three simulations are using the same computational model for increasing frequency, the resolutions for the three cases are different. The resolution for the three frequencies are and 6 respectively for 1,2 and 3. As a result, it is likely that the discrepancy for is due to relatively low resolution. To further examine the influence of resolution two more simulations are performed for rotating dipoles at $\quad 1$, one with a $p p w$ of 6 and one with a of 12 . The number of equivalent sources is chosen to be $1 / 3$ of the number of control points following Tinetti and Dunn ${ }^{12}$ for traditional ESM. The results, as well as analytical solution and CESM prediction with a $p p w$ of 18, are shown in Figure 13. From the figure it can be seen that with a $p p w$ of 6 , the simulation result is unsatisfactory, especially in the shadow zone. With a $p p w$ of 12 , the predicted directivity pattern is almost coincident with the analytical solution, except a $2 \mathrm{~dB}$ under-prediction of total SPL at the second peak $\left({ }^{\circ}\right)$ and the third valley $\left(\phi=210^{\circ}\right)$ trough in the shadow zone. As expected the best results are obtained with a of 18 , at a cost of consuming 10 times of computation time comparing to the computation with 12 points per wavelength. Meanwhile, comparison of Figures 12 and 13 shows that, with a of 6, at the predictions are much more accurate than those at 1 . It indicates that as the frequency increases, the accuracy of prediction improves with the same level of resolution, as was found by Tinetti and Dunn ${ }^{12}$ for the traditional ESM. This suggests that for scattering problems with higher frequencies, the computational cost could be relieved by using less number of points per wavelength.

\section{Summary}

For sound scattering problems, a traditional equivalent source method with real equivalent sources has difficulties in simulating scattering off thin objects or objects with thin, sharp edges. A real equivalent source surface has to be placed interior to the scattering surface with a certain distance. Towards a thin, sharp edge such as the trailing edge of an airfoil, the thickness of the scattering object may be too small to allow the placement of a suitable source. Under such circumstances traditional equivalent source methods may fail to capture the scattering effects off the thin, sharp edge. This disadvantage of traditional equivalent source method is overcome in this study with a new, complex equivalent source method.

In this work a new, complex equivalent source method is developed to simulate the scattering effects of a realistic geometry with sharp edges. This method replaces the real equivalent sources in a traditional equivalent source method with complex equivalent sources which have different properties in real space. Such properties allow complex equivalent monopoles to be placed close to a scattering surface. Therefore, the scattering effects of thin, sharp edges like trailing edges of lifting surfaces can be captured with sufficient accuracy.

Benchmark test case studies of the new complex equivalent method have been performed. They included simulations of scattering by 2D cylinders, 2D bodies with elliptic and rectangular cross-sections, and 2D airfoils. Simulations are also performed of scattering of rotating sources by a 3D cylinder of finite length. Results have shown good agreement with analytical solutions. For test cases without analytical solutions, the results are qualitatively correct as well. Results for scattering by an airfoil which has a thin, sharp trailing edge have shown that the complex equivalent source method behaves better than the traditional equivalent source method in predicting the scattering effects by sharp edges.

Further studies are necessary to allow the new method to be used with confidence. Firstly, a systematic study of the influence by the imaginary part of the complex sources should be performed, the outcome of which are important to determine the appropriate locations of complex equivalent sources. Secondly, the effects of flow non-uniformities are ignored. This might be partly solved by integrating non-uniform potential flow theory as done by Astley and Bain ${ }^{20}$. Thirdly, at high frequencies the computation costs could become excessive for a fullscale airframe. To overcome this, the FMM will be implemented in future version of ESSC to accelerate the solution. 


\section{Acknowledgement}

Yu Hou would like to thank China Scholarship Council (CSC) and University of Southampton for providing financial support for this research.

\section{References}

${ }^{1}$ Flightpath 2050 Europe's Vision for Aviation: Report of the High Level Group Research, European Commission, 2011.

${ }^{2}$ McAlpine, A. and Kingan, Michael J., Far-field Sound Radiation due to an Installed Open Rotor, International Journal of Aeroacoustics, Vol. 11, No. 2, 2012, pp. 213-245.

${ }^{3}$ Amiet, R. K., Unified Aeroacoustics Analysis for High Speed Turboprop Aerodynamics and Noise Volume II Development of Theory for Wing Shielding, NASA CR-185192, 1991.

${ }^{4}$ Mechel, F. P., Formulas of Acoustics, $2^{\text {nd }}$ edition, Springer, 2008.

${ }^{5}$ Liu, Y., Fast Multipole Boundary Element Method: Theory and Applications in Engineering, Cambridge University Press, Cambridge, 2009.

${ }^{6}$ Gumerov, N. A., and Duraiswami, R., Fast Multipole Methods for the Helmholtz Equation in Three Dimensions, $1^{\text {st }}$ edition, Oxford, UK, Elsevier, 2004.

${ }^{7}$ Ochmann, M., The Source Simulation Technique for Acoustic Radiation Problems, Acta Acustica united with Acustica, Vol. 81, No. 6, 1995, pp. 512-527.

${ }^{8}$ Koopmann, G. H., Song, L. and Fahnline, J. B., A Method for Computing Acoustic Fields Based on the Principle of Wave Superposition, Journal of Acoustic Society of America, Vol. 86, No. 6, 1989, pp.2433-2438.

${ }^{9}$ Miller, R. D. and Myer, T. Jr., Comparison between the Boundary Element Method and the Wave Superposition Approach for the Analysis of the Scattered Fields from Rigid Bodies and Elastic Shells, Journal of Acoustic Society of America, Vol. 89, No. 5, 1991, pp. 2185-2196.

${ }^{10}$ Jeans, R. and Mathews, I. C., The Wave Superposition Method as a Robust Technique for Computing Acoustic Fields, Journal of Acoustic Society of America, Vol. 92, No. 2, 1992, pp. 1156-1166.

${ }^{11}$ Dunn. M. H., and Tinetti, A. F., Aeroacoustic Scattering via the Equivalent Source Method, AIAA 2004-2937, 2004.

${ }^{12}$ Tinetti, A. F. and Dunn, M. H., The Fast Scattering Code (FSC): Validation Studies and Program Guidelines, NASA CR-2011-217158, 2011.

${ }^{13}$ Lee, S., Brentner, K. S. and Morris, P. J., Acoustic Scattering in the Time Domain Using an Equivalent Source Method, AIAA Journal, Vol. 48, No. 12, 2010, pp. 2772-2780.

${ }^{14}$ Lee, S., Brentner, K. S. and Morris, P. J., Assessment of Time-Domain Equivalent Source Method for Acoustic Scattering, AIAA Journal Vol. 49, No. 9, 2011, pp. 1897-1906.

${ }^{15}$ Gounot, Y. J. R. and Musafir, R. E., Simulation of Scattered Fields: Some Guidelines for the Equivalent Source Method, Journal of Sound and Vibration, Vol. 330, 2011, pp. 3698-3709.

${ }^{16}$ Blokhintsev, D. I., Acoustics of a Nonhomogeneous Moving Medium, NACA TM-1339, 1956 (Translation of the Russian original).

${ }^{17}$ Ochmann, M., Complex Source Point Method for Computing the Green's Function over an Arbitrary Impedance Plane, Proceedings of the NOVEM 2005, St. Raphael, France, 2005.

${ }^{18}$ Ochmann, M. and Piscoya, R., The Source Simulation Technique with Complex Source Points for Computing Acoustic Radiation Problems, ICSV13, Vienna, 2006.

${ }^{19}$ Dunn, M. H., Application of Fast Multipole Methods to the NASA Fast Scattering Code, AIAA 2008-2875, 2008.

${ }^{20}$ Astley, R. J. and Bain, J. G. , A Three-Dimensional Boundary Element Scheme for Acoustic Radiation in Low Mach Number Flows, Journal of Sound and Vibration, Vol. 109, 1986, pp. 445-465. 